\title{
Contamination of urine specimens did not differ with collection technique in women with acute dysuria
}

\author{
Lifshitz E, Kramer L. Outpatient urine culture. Does collection technique matter? Arch Intern Med 2000 Sep \\ 11;160:2537-40.
}
QUESTION: In women with acute dysuria, does contamination of urine specimens differ with collection technique (midstream, midstream plus vaginal tampon, or non-midstream with no cleansing)?

\author{
Design \\ Randomised \{allocation not concealed*\}†, blinded (out- \\ come assessors),* controlled trial.
}

\section{Setting}

An outpatient clinic for students at Rutgers University, New Jersey, USA.

\section{Patients}

242 consecutive women (mean age 21 y) who were mostly undergraduates and had symptoms suggestive of cystitis. Exclusion criteria were antibiotic use, use of urethral instrumentation in the previous 7 days, or known urological abnormality or nephrolithiasis. Follow up was complete.

\section{Intervention}

84 women were allocated to midstream collection and were instructed to cleanse the perineum with a bactericidal wipe by wiping from front to rear; spread the labia; discard the first urine output; and then collect the midstream specimen in a clean, non-sterile container. 81 women were allocated to midstream collection plus a vaginal tampon. They were given the same instructions as the midstream group but were also instructed to insert a vaginal tampon before collection of the specimen. $\{3$ or 4$\} \nmid$ women unable or unwilling to use a tampon were reallocated to the midstream group. 77 women were allocated to the non-midstream with no cleansing group and were instructed to urinate into a clean, non-sterile container without cleansing the perineum or discarding the first urine output.

\section{Main outcome measures}

Contamination of urine specimens assessed by microbial composition of cultures. Samples were considered contaminated if they contained Enterococcus, Streptococcus viridans, Staphylococcus aureus, $S$ epidermidis, or a mixed culture of $\geqslant 2$ organisms.

\section{Main results}

The midstream (32\%), midstream plus vaginal tampon $(31 \%)$, and non-midstream with no cleansing (29\%) groups did not differ for rate of contaminated specimens $(\mathrm{p}=0.82)$. When the 2 interventions were analysed together as 1 group and compared with the no cleansing group, the lack of difference remained $(\mathrm{p}=0.65)$.

\section{Conclusion}

In women with acute dysuria, contamination of urine specimens did not differ with collection technique (midstream, midstream plus vaginal tampon, or nonmidstream with no cleansing).

*See glossary.

$\dagger$ Information provided by author.

\section{COMMENTARY}

In a letter to the Lancet in 1979, ${ }^{1} 2$ British general practitioners asked whether traditional methods of collecting urine for culture were a necessary ritual. They presented data from their own practice indicating that rates of contamination in specimens collected with or without the usual precautions were no different.

20 years later, Lifshitz and Kramer have confirmed that the usual contortions associated with traditional methods of collecting a midstream urine specimen are unnecessary. Equivalent results are obtained by simply requesting the patient to urinate into a clean container.

Current guidelines aim to decrease or eliminate the use of urine culture as a guide to the diagnosis and treatment of acute urinary tract infection (UTI). ${ }^{2}$ On the basis of a cost utility analysis of office based treatment strategies, Barry et al concluded that the preferred strategy was one of empiric treatment without urine culture. ${ }^{3} \mathrm{~A}$ trial in 24 primary care clinics with almost 4000 patients indicated that treatment based on advice and prescription over the telephone, instead of an office visit, was effective in decreasing laboratory use and overall costs while maintaining or improving the quality of patient care. ${ }^{4}$

What is the bottom line? In acute, uncomplicated UTIs, urine culture is unnecessary. In other circumstances, such as chronic or recurrent infection, urine culture may be indicated, in which case a simple urine sample in a clean container is adequate.

Anthony S Dixon, MB, ChB University of Hong Kong Hong Kong, China

1 Morris RW, Watts MR, Reeves DS. Perineal cleansing before midstream urine, a necessary ritual. Lancet 1979;2:158-9.

2 Houston M. Uncomplicated urinary tract infection in women. Diagnostic and therapeutic recommendations by the Institute for Clinical Systems Integration. Postgrad Med 1999;105:181-3,187-8.

3 Barry HC, Ebell MH, Hickner J. Evaluation of suspected urinary tract infection in ambulatory women: a cost-utility analysis of office-based strategies. J Fam Pract 1997;44:4960.

4 Saint S, Scholes D, Fihn SD, et al. The effectiveness of a clinical practice guideline for the management of presumed uncomplicated urinary tract infection in women. Am J Med 1999;106:636-41.
Source of funding: no external funding.

For correspondence: Dr E Lifshitz, Rutgers University Health Services, 110 Hospital Road, Piscataway, NJ 08854, USA.Fax + 7324453725 .

A modified version of this abstract appears in Evidence-Based Nursing. 